\title{
Tubular ectasia of the rete testis (TERT). Differential diagnosis of cystic testicular disorders
}

\author{
Alois Mahlknecht ${ }^{1}$, Peter Mahlknecht ${ }^{2}$, Mohamad Fallaha ${ }^{1}$, Anton Wieser ${ }^{3}$ \\ ${ }^{1}$ Department of Urology, General Hospital Meran, Italy; \\ ${ }^{2}$ Department for Evidence-based Medicine and Clinical Epidemiology, Danube University Krems, Austria; \\ ${ }^{3}$ Department of Radiology, General Hospital Meran, Italy.
}

\begin{abstract}
Summary The ultrasound scan plays an essential role in the urological-andrological diagnosis. High-resolution transducers $(8-15 \mathrm{MHz})$ make it possible to prove increasingly small changes. The assessment of cystic masses in the testis can also be difficult for experienced doctors. However, a precise diagnosis is crucial for the patient to avoid further invasive diagnostics and therapy. The differential diagnosis of benign intra-testicular cystic lesions include the tubular ectasia of the rete testis (TERT), the cystic dysplasia, epidermoid cysts, dermoid cysts, simple testicular cysts and cysts of the tunica albuginea. Malign testicular tumours with cystic changes are particularly the mature teratoma, carcinomas of the epididymis and metastasis. The following overview shows different sonographic images and interpretations with a particular focus on TERT.
\end{abstract}

KEY WORDS: Tubular ectasia of the rete testis (TERT); Testicular cysts; Tubular ectasia; Cystic dysplasia.

Submitted 15 September 2014; Accepted 30 November 2014

\section{INTRODUCTION}

With the almost routine use of scrotal sonography, cystic masses are increasingly diagnosed; however, the clinical significance is often unclear. In general formations of cysts of the testicle are difficult to evaluate, because intratesticular cavities can also be typically formed by teratomatous germ cell tumours (1). The magnetic resonance imaging (MRI) can be used for further clarification; in contrast to the sonography the MRI has the advantage that a more exact tissue differentiation obtained through the evaluation of several signal qualities (2). The indication for diagnostic operative exploration is frequently made resulting in the demonstration of cystic lesions. Only in recent years it has been shown that most of the cystic cavities in the testicle have a benign character and that under no circumstances surgery is required (3). In the following, the pathogenesis and the sonographic image of TERT will be described and the differential diagnosis in comparison with other cystic formations in the testis will be illustrated.

\section{TUBULAR ECTASIA OF THE RETE TESTIS (TERT)}

The Tubular Ectasia of the Rete Testis (TERT) is defined as an intra-testicular area outgoing from the testicular hilum, which contains numerous tight and anechoic (cystic) lesions $(4,5)$. The typical sonographic image arises from the enlargement of the numerous canaliculi of the rete testis.

\section{Pathogenesis of TERT}

The etiology of TERT has not been definitely clarified. Nistal et al. (6) consider four possible causes:

- a mechanical obstruction,

- primary congenital deformity,

- ischaemic degeneration of the efferent ducts (from the rete testis to the epididymis) and

- hormonal mechanisms, particularly an androgen deficiency.

The vascular hypothesis is the most widely accepted one. The artery which supplies the caput epididymis is a very thin branch of the testicle's main artery; therefore, arteriosclerotic changes in old age can lead to circulatory disorders. According to this, TERT mainly appears at an advanced age, normally between the age of 54 and 64 . Because of reduced perfusion there is a degeneration of the metabolically active tissue in the region of the rete testis. Physiologically, $90 \%$ of the seminal fluid is reabsorbed and only $10 \%$ of the quantity of secretion is transmitted into the epididymis through the efferent tubules. As a consequence of the degeneration, the process of reabsorption can't occur anymore and thus the prime seminal fluid accumulates in the branched canal system of the rete testis.

The androgen deficiency, which appears usually from the age of 50, applies to the incidence of TERT at an advanced age as well. The androgen deficiency leads to an atrophy of the epididymal duct with following mechanical outflow obstruction of the seminal fluid. An interaction of vascular and endocrinal factors is also conceivable in the development of TERT.

However, the frequent association of TERT with a spermatocele in the region of the epididymis militates for the mechanically caused development of TERT. The term 
"intra-testicular spermatocele" has therefore been coined by Davis (7). Some of the cases of TERT described in the literature show a history of anamnestic surgery like a hernioplasty or a vasectomy; as well pre-existing conditions, like epididymitis are often present.

\section{Sonographic aspects of TERT (Figures 1, 2)}

With the improvement of the sonographic technique, cystic changes in the testicle are diagnosed as an incidental finding with increasing frequency. First case reports which today correspond to TERT were published in 1987 (8), among which the lesions were considered to be a curiosity without systematic classification of the result. Cystic masses can be shown with high diagnostic certainty only with high-resolution sound frequency of 7.5-12.0 MHz. The first systematic description of TERT as a distinct entity was given in 1992 (3). The benign character of TERT has been clearly proved by the evaluation of 31 cases. The term "TERT" was proposed by Tatar et al. (9) in 1993 and nowadays is generally recognised (10).

\section{Figures 1, 2.}

Typical findings of a TERT: anechoic areas differing in size in the region of rete testis which fill the major part of the testis and therefore suppress the remaining parenchyma; 58-year-old patient.

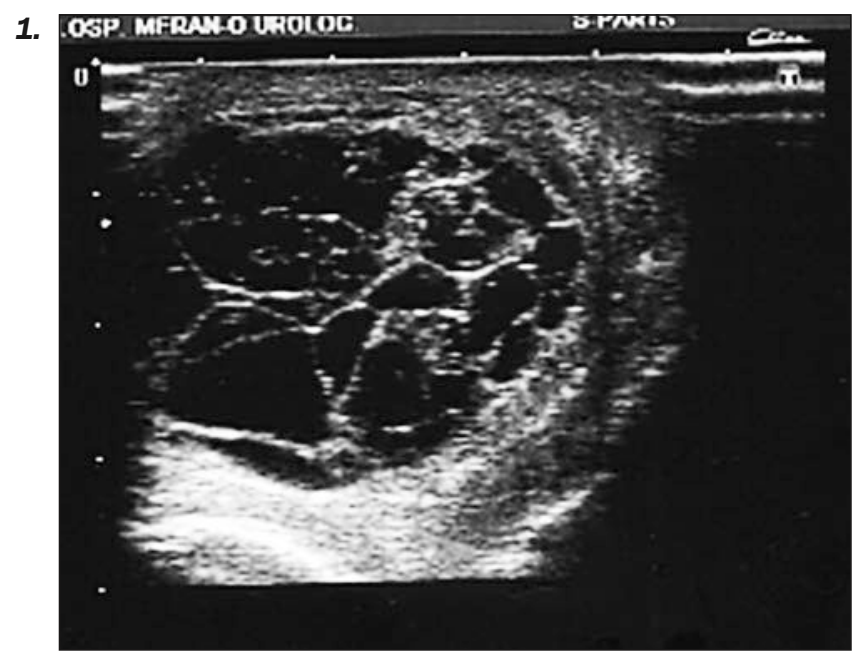

2.

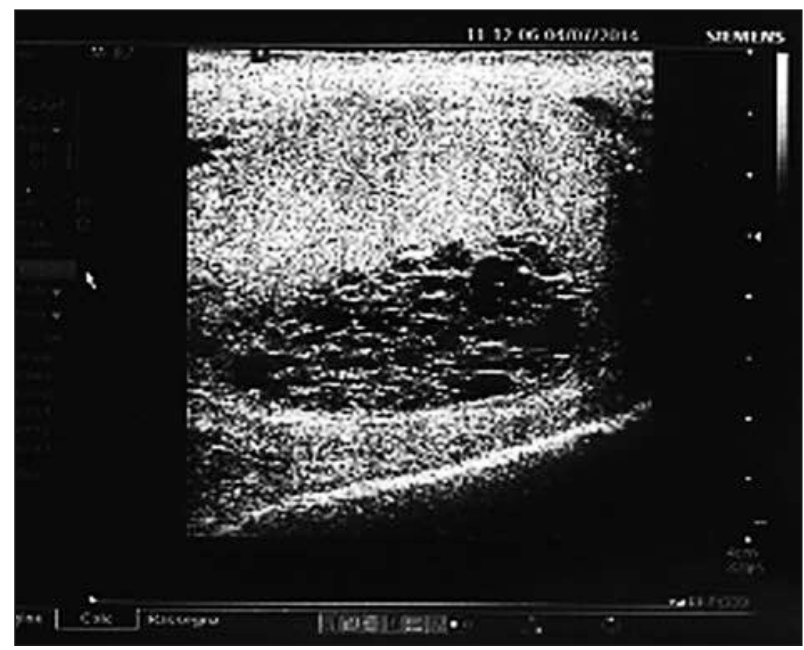

The sonographic image of TERT consists of an area outgoing from the mediastinum testis with numerous tight, anechoic and rounded nodules which show a short-path dorsal acoustic enhancement.

The anechoic single lesions are mostly smaller than 3 $\mathrm{mm}$; however, also bigger ones can exist.

The MRI can be used for further diagnosis; certainly, the costs are higher and it is more time-consuming. Nevertheless, the MRI has the advantage that the examinations can be carried out with the application of contrast agents (gadolinium), and the organs can therefore be examined in several signal qualities. TERT was first examined with MRI in 1993 (9).

\section{Therapy of TERT}

The benign character of TERT is well assured. Therefore, a specific therapy, and especially surgery, is not required.

\section{Differential diagnosis}

1) The most important differential diagnosis of TERT is the cystic teratoma. About $10 \%$ of all germ cell tumours contain teratomatous content. But in these cases - in contrast to TERT - there is mostly an increase in size of the testicle or a palpable tumour. Sonographically, a solid content is almost always provable. The eccentric, peripheral localisation of an intra-testicular cyst militates against TERT, which is always located at the mediastinum testis. In those cases, the implementation of MRI and a surgical exploration are indicated.

2) The cystic transformation of the rete testis has also sonographic similarities with TERT, but it is a congenital deformity. Pathogenetically, it concerns a developmental disorder in which the canaliculi of the mesonephros have no connection to the gonad blastema. Since the canaliculi of the mesonephros will develop into the efferent ducts, the outflow for the testicular secretion towards the epididymis is missing (11). Consequently, it comes to impoundment of the testicular secretion. Clinically, a swelling of the testicles results; sonographically, there is an area centrally located in the testicles with numerous anechoic lesions. These lesions are usually visible unilaterally. The cystic transformation of the rete testis is very often associated to ipsilateral renal agenesis or other renal dysplasias.

3) Another differential diagnosis of TERT is the simple testicular cyst which can be randomly found. Sonographically they appear as round and anechoic, mostly solitary cyst with a size of $0.2-2.0 \mathrm{~cm}$. The pathogenesis is unknown. With a higher resolution an echoic margin, which surrounds the cyst, appears. In this case, it concerns a testicular parenchyma.

4) Cysts of the tunica albuginea are mostly solitary and exhibit generally a size of $5-8 \mathrm{~mm}$. The cause is unknown and the result is benign. A posttraumatic or postinfectious genesis, as well as a development from embryonic structures is possible. Cysts of the tunica albuginea were first classified as a separate entity by 
Figure 3.

Large intratesticular cyst.

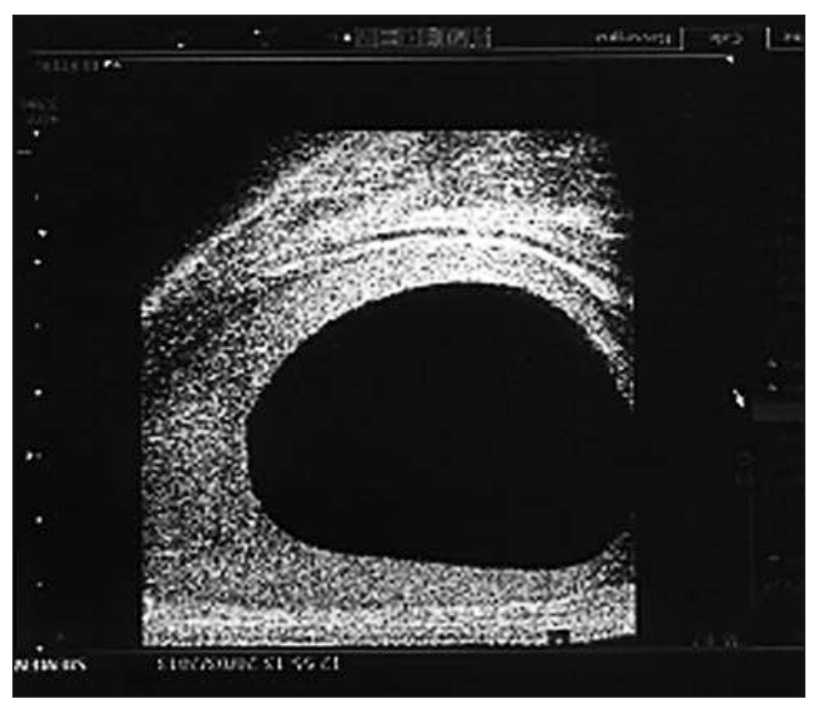

Frater in 1929 (12). Sonographically, they appear localized and anechoic as well. They have a thin margin and are adherent to the tunica albuginea (Figure 3 ).

5) The epidermoid cyst is a benign cystic mass in the testis which consists of a fibrous facing, inside of a keratinised squamous epithelium and at the centre of cell debris. The cysts can reach a size of several centimetres. They were first described by Dockerty and Priestly in 1942 (13). Epidermoid cysts appear in adolescence and in young adulthood, the typical age of testicular tumours. A highly echogenic margin is the sonographic characteristic. It corresponds to the structure of the margin of the cyst consisting of fibrous material with partial calcification. Alternating hypoechogenic and hyperechogenic rings ("onion ring" pattern) represent strata of compact keratin and loosely spread desquamated cells. In contrast to TERT, weak echo reflexes can occur in the centre of epidermoid cysts; these weak echo reflexes are caused by the viscous cyst content. As a result, the epidermoid cyst sonographically appears as a target.

\section{CONCLUSION FOR PRACTICE}

Cystic masses of the testicle can be benign or malignant. Concerning the establishment of the further treatment, a differentiation of these two entities is significant for the patient and the physician in charge. Therefore, the scrotal ultrasonography is absolutely necessary. If a benign cystic mass of the testis is diagnosed, an organ sparing therapy or a follow-up procedure can be recommended in most cases.

\section{REFERENCES}

1. Connolly SS, D`Arcy FT, Gough N, et al Carefully selected intratesticular lesions can be safely managed with serial ultrasonography. BJU Int. 2006; 98: i1005-1007.

2. Nagler-Reus M, Guhl L, Volz C, et al. Magnetresonanztomographie des Skrotums. Erfahrungen an 129 Patienten. Radiologe 1995; 3:494-503.

3. Brown DL, Benson CB, Doherty FJ, et al Cystic testicular mass caused by dilated rete testis: sonographic findings in 31 cases. Am J Roentgenol. 1992; 15:1257-1259.

4. Older RA, Watson LR. Tubular ectasia of the rete testis: a benign condition with a sonographic appearance that may be misinterpreted as malignant. J Urol. 1994; 152:477-478.

5. Rouviere O, Bouvier R, Pangaud C, et al. Tubular ectasia of the rete testis: a potential pitfall in scrotal imaging. Eur Radiol. 1999; 9:1862-1868

6. Nistal M, Mate A, Paniagua R. Cystic transformation of the rete testis. Am J Surg Pathol. 1996; 20:1231-1239.

7. Davis RS. Intratesticular spermatocele. Urology. 1998; 51:167-169.

8. Gooding GA, Leonhardt W, Stein R. Testicular cysts: US findings. Radiology. 1987; 163:537-538.

9. Tartar VM, Trambert MA, Balsara ZN, Mattrey RF. Tubular ectasia of the testicle: sonographic anMR imaging appearance. Am J Roentgenol. 1993; 160:539-542.

10. Pavlica P, Ramini R, Barozzi L Tubular ectasia of the rete testis. The ecographic aspects. Radiol Med. 1994; 87:493-497.

11. Eberli D, Gretener H, Dommann-Scherrer C, et al. Cystic displasia of the testis: a very rare paediatric tumor of the testis. Urol Int. 2002; 69:1-6.

12. Frater K. Cysts of the tunica albuginea (cysts from the testis). J Urol. 1929; 21:135-140.

13. Dockerty MB, Priestly JT. Dermoid cysts of the testis. J Urol. 1942; 48:392-400.

\section{Correspondence}

Mahlknecht Alois, MD

alois.mahlknecht@asbmeran-o.it

Mohamad Fallaha, MD

General Hospital "Franz Tappeiner"

V. Rossini 5 - 39012 Merano, Italy

\section{Peter Mahlknecht, MD}

Department for Evidence-based Medicine and Clinical Epidemiology

Danube University Krems, Krems, Austria

Anton Wieser, MD

Department of Radiology, General Hospital

Merano, Italy 